\title{
STUDY OF CONFIGURATION DEPENDENT RESOLUTION OF 3-D BURRIED CONDUCTORS
}

\author{
E. Auken ${ }^{\dagger}$, G. M. Hoversten* and H. F. Morrison* \\ 'Department of Geology and Geotechnical Engineering, \\ The Danish Technical University, DK-2800 Lyngby, Denmark \\ *Department of Material Science and Mineral Engineering, \\ University of California, Berkeley, CA 94720, USA
}

\begin{abstract}
The electromagnetic response of a buried conductor is a complex function of the currents induced and channeled into the conductor from the currents flowing in the background media. The ration between the channeling and induced currents is basically determined by the currents in the background half-space that are available for channeling and on the magnetic field on the conductor. In order to "see" the target the secondary fields must exceed some threshold value which is dependent on the layered background response and on the source receiver configuration.

To compare objectively the detection efficiency of various source receiver configurations we express the responses in terms of the ratio of the secondary field to the field from the background media. This ratio is called the anomaly index, AI. Using the AI to compare different source receiver configurations energizing the same target provides a completely general way to compare the performance of any EM system in either time or frequency domain or to compare between the two domains.

The concept of the AI is used to compare normal incident plane waves, a infinite line, a grounded electric dipole (of variable length) and a horizontal loop (of variable side length) over a thin rectangular sheet buried in a conductive half-space. The $\mathrm{AI}$ is computed in both time and frequency domain for a whole range of source-receiver parameters.

In general we find that the AI for EM systems working in the time domain is larger than for systems working in the frequency domain. The main reason for this is that frequency domain systems has to "see" the target in the presence of the primary fields from the source whereas the primary fields by nature is absent for time domain systems. Furthermore, it is found that for a vertical sheet, which is used in this demonstration, the AI for the horizontal transient fields from the horizontal loop source is substantially larger than for the vertical fields (which is the response usually measured).
\end{abstract}

\section{INTRODUCTION}

In the literature there has been exhaustive studies of the basic response of a variety of subsurface targets in both time and frequency domain. An excellent review is given in the SEG volume "Electromagnetic methods in Applied Geophysics" (Nabighian (ed.), 1988) where a logical grouping has been in chapters dedicated to sounding methods (determination of conductivity vs. depth), profiling methods (with a variety of source-receiver configurations) and finally to uniform field methods such as magnetotelluric and VLF. By and large these studies have focused on specific systems in common use for exploration but there has been little comparison of the relative response of different systems to common targets.

In this presentation we describe a generalized approach for comparing the response of conductive bodies to excitation by dipole, line and uniform incident fields in both time and 
frequency domain. These sources are (among others) charatized by different geometrical fall off rates of their fields.

The response of the target is superimposed on the response of the host or the background half-space. The target response itself is made up of a combination of current channeling (currents induced in the background half-space being concentrated in the target) and currents induced in the target (inductive currents) (Walker and West, 1992). The channeling currents are proportional to the tangential electric field and the inductive currents are proportional to the incident magnetic field normal to the surface. In a practical situation the background, inductive and channeling currents can not be separated and they are in close interaction. Though, the degree of channeling and inductive current flow is very dependent on the electromagnetic source and also the background response varies because of the source dependent geometrical fall off of the primary field.

A practical exploration system will "see" the response of a buried conductor if the response of the target rises above some threshold fraction of the response from the background medium. The threshold fraction is system dependent and is given by the exploration system and on the background noise in the sounding environment. Noise is in this case electromagnetic background noise as well as geological noise (geological complexity not included in the model). Comparing different source-receiver configurations in terms of an Anomaly Index (AI), defined as the ration of the secondary field to the amplitude or modulus of the same field component over the half-space, transfer the influence of source moment and noise to a later stage in the experimental design and the comparison can be carried through without being caught into practical measurement considerations. To use the AI in a practical application one would have to use the layered response to determine the source moment for the given configuration that produces a field strength in the desired direction that can be measured with the available receiver. Then the AI yields the fraction of the field that a given target will yield for the given source type.

\section{AI FOR A THIN SHEET MODEL - RESULTS}

The model considered in this example is a single vertical sheet with a conductivitythickness product of $1000 \mathrm{~S}$, strike and depth extent is $200 \mathrm{~m}$ by $100 \mathrm{~m}$ and the conductivity of the half-space is $100 \Omega \mathrm{m}$. The depth to the top of the sheet is $100 \mathrm{~m}$ and the center of the sheet is offset from the origin by $100 \mathrm{~m}$ in the $\mathrm{x}$-direction. The sources, vertical magnetic
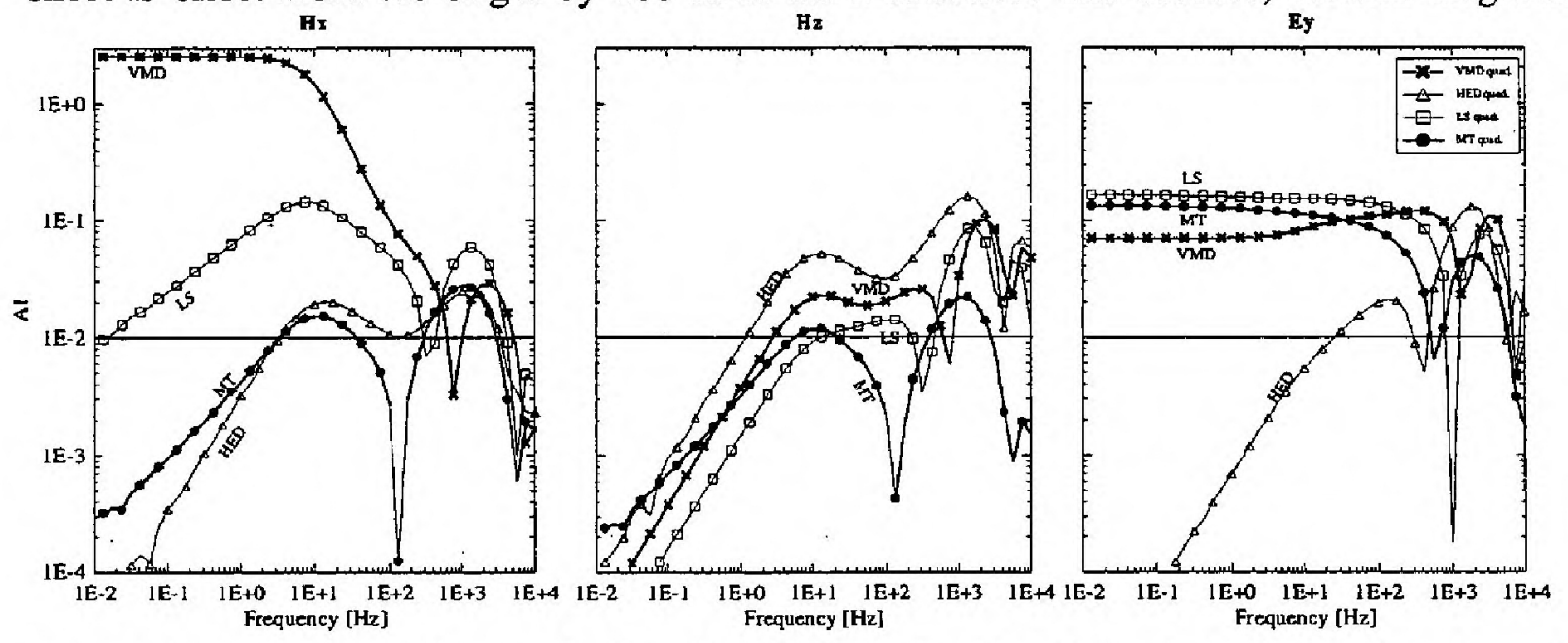

Figure 1. The AI for the quadrature $H_{x}, H_{z}$ and the $E_{y}$ fields plotted as a function of frequency. The line at $1 . \mathrm{e}^{-02}$ indicate a $1 \% \mathrm{AI}$. The $\mathrm{H}_{\mathrm{z}}$ fields for the plane wave source are normalized with the amplitude of $\mathrm{H}_{\mathrm{x}}$. 
dipole (VMD), grounded electric dipole (y-directed) (HED), infinite line source (y-directed) (LS) and plane waves (MT) are located at the origin.

Figure 1 shows the frequency domain quadrature AIs for the $H_{x}, H_{z}$ and the $E_{y}$ components plotted as a function of frequency. They are presented for those receiver sites where AI maximizes; directly above the sheet for the $\mathrm{H}_{\mathrm{x}}$ component and offset to the right of the sheet for the $\mathrm{H}_{\mathrm{z}}$ and $\mathrm{E}_{\mathrm{y}}$ components. As seen in Figure 1 the VMD produces the largest $\mathrm{AI}$ for the $\mathrm{H}_{\mathrm{x}}$ component especially at low frequencies. The HED produces the highest AI in the vertical component and the LS (and MT) produces the highest AI in the electric field component parallel to strike $\left(E_{y}\right)$. Many of the curves have obviously sign reversals above 100 $\mathrm{H}_{\mathrm{z}}$ and often the AIs for different source-receiver configurations change sign on either side of these reversals. The fundamental reasons for this and the other apparently complex AIs in Figure 1 lie in the interplay of source field fall-off, channeling vs. inductive response and secondary field fall off from the sheet. By studying many plots of AIs for different sourcereceivers configurations vs. targets and examine the currents flowing in the half-space it is clear that the AIs is determined by how fast the layered fields fall off with distance compared to the amplitude of the scattering currents which generate the secondary field (Auken et al., 1996).

Expressing the $\mathrm{AI}$ in the time domain results in the plots shown in Figure. 2. The advantage of working in the time domain, measuring fields in the absence of the primary field, is clearly revealed by these AIs, especially from the VMD and the HED. At times beyond 1 ms the AIs is as much as 100 times the maximum frequency domain AIs. It is interesting to note that the AIs for the horizontal component from the VMD is more than a factor of 10 larger than the AIs for the vertical component and that the $\mathrm{H}_{\mathrm{z}}$ component from the HED produces the largest $\mathrm{AI}$ at all.
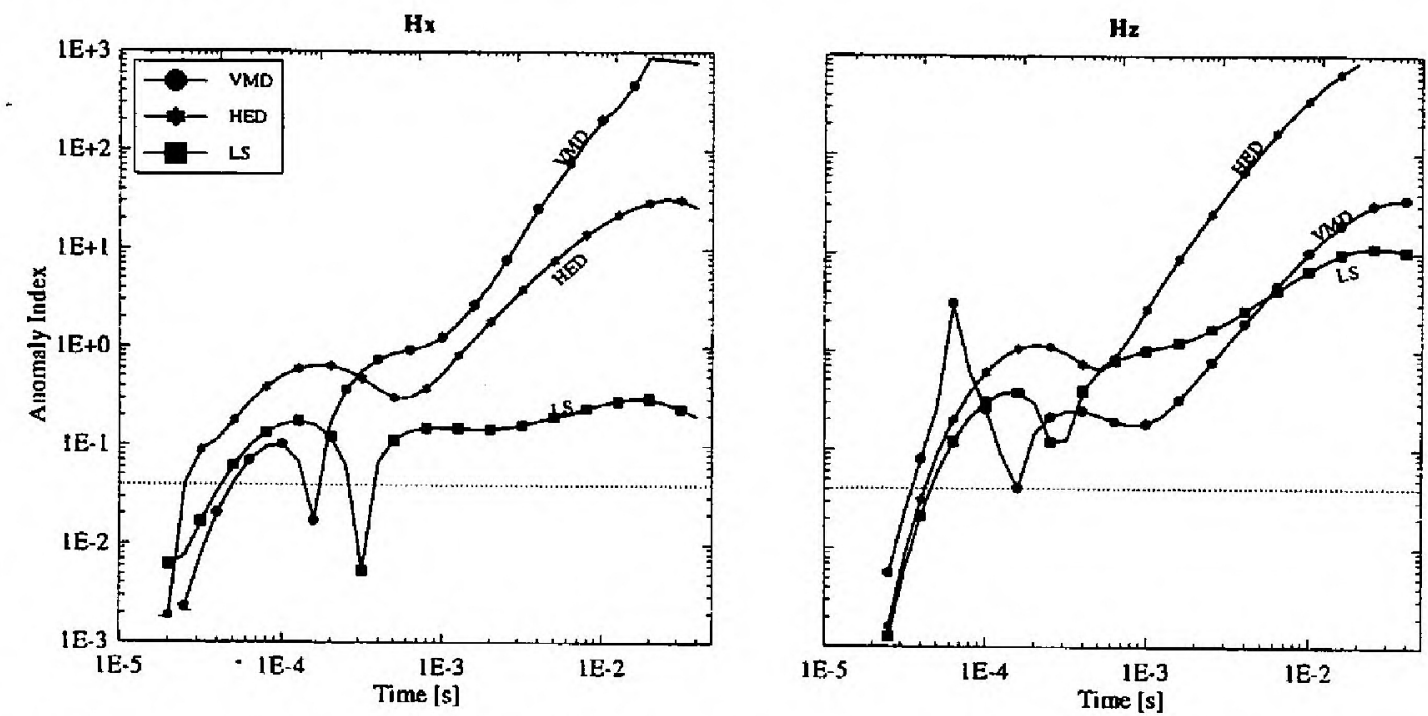

Figure 2. The time domain AI. The line at $3 . e^{-02}$ indicate a $3 \% \mathrm{AI}$.

Finally we will show an example of an analyze of a transient loop-dipole system (Figure 3). The system is measuring the transient $H_{x}$ and $H_{z}$ components. As the variable parameters in this comparison is large - source-receiver separation, loop side length, measurement position relative to the sheet and measurement time - we have chosen to express the $\mathrm{AI}$ as a $\mathrm{AI}$-peak to peak $\left(\mathrm{AI}_{\mathrm{pp}}\right)$. The $\mathrm{AI}_{\mathrm{pp}}$ is the maximum $\mathrm{AI}$ for a given source-receiver combination, loop side length, measurement position and measurement time. I.e. $\mathrm{AI}_{\mathrm{pp}}$ is the maximum AI the source-receiver combination can yield at any instance for the target. 
The contours of the $\mathrm{AI}_{\mathrm{pp}}$ in Figure 3 covers the cases of the central loop configuration which has a separation of $0 \mathrm{~m}$, the out of loop configuration for which the separation is larger than half the loop size and finally the VMD for separations larger than two to three times the loop size. The contour interval is equally spaced in log space and each contour is based on 24 model calculations. In Figure $3 \mathrm{~b}$ the $\mathrm{AI}_{\mathrm{pp}}$ maximizes for a loop size and a separation approximately equal to half the depth to the sheet. When the transient horizontal component is measured the maximum $\mathrm{AI}_{\mathrm{pp}}$ is obtained at a separation on the order of the depth to the sheet and for a loop size of half the depth to the sheet. Furthermore, the $\mathrm{AI}_{\mathrm{pp}}$ is more than a factor of 20 larger for the horizontal component compared to the vertical component.
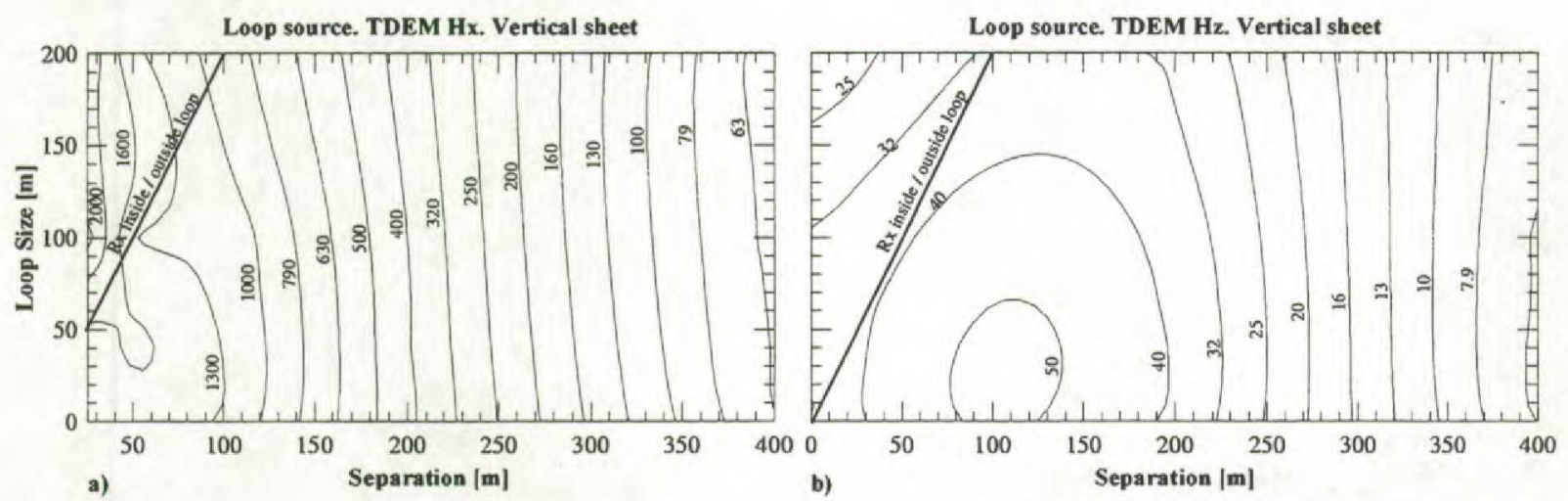

Figure 3. Contour of $\mathrm{AI}_{\mathrm{pp}}(\mathrm{t})$ for the $\mathrm{H}_{\mathrm{x}}$ and $\mathrm{H}_{\mathrm{z}}$ fields as a function of separation and loop size.

\section{CONCLUSION AND DISCUSSION}

Two important results of this study are the large AI for the transient $\mathrm{H}_{\mathrm{x}}$ from the loop source and $\mathrm{H}_{\mathrm{z}}$ from the HED source (figures are not shown here). For the loop source the $\mathrm{AI}$ is a factor of 10 to 20 larger when measuring $\mathrm{H}_{x}$ compared to $\mathrm{H}_{\mathrm{z}}$. Measuring the transient $\mathrm{H}_{\mathrm{z}}$ field from a HED source yields an AI a factor of 20 to 40 greater than the $\mathrm{H}_{\mathrm{z}} \mathrm{AI}$ for the loop source. Thus, much smaller anomalies can be detected using a TDEM system measuring the horizontal field from a loop source or the vertical field from a HED source. Noting that the loop source is very practical to handle in the field the only drawback on measuring horizontal components compared to vertical components is a substantially larger natural noise level in the horizontal components.

In the analysis the frequency domain systems have a much smaller AI compared to the TDEM systems. This is due to the fact that FDEM systems measures the target response in the presence of the primary field and that the noise level due to orientation errors of the coils is high (McCracken, 1980). The only exception from this conclusion is the case when very large penetration depth is required as in e.g. oil exploration. For these situations a controlled source of a sufficient strength is not very practical in the field and plane wave methods are a desirable alternative to time domain methods.

\section{REFERENCES}

Auken,E., Hoversten,G.M. and Morrison,H.F., 1996, The role of source dimensionally in the electromagnetic response of buried conductors, Paper submitted to Geophysics

Nabighian, M. N., [Ed], 1988, Electromagnetic methods in applied geophysics, in Electromagnetic methods in applied geophysics, 1: Soc. Expl. Geophys., 503 pp

McCracken, K.G., Hohmann, G.W. and Oristaglio, M.L. 1980, Why time domain, Bull Aust Soc. Explor. Geophys., 11, 176 - 179

Walker, P. W. and West, G. F., 1992, Parametric estimators for current excitation on a thin plate, Geophysics, 57, 766-773 